This is a postprint version of the following published document:

Antonelli, C., Serrano, B., Baselga, J., Ozisik, R.

\& Cabanelas, J. C. (2014). Interfacial

characterization of epoxy/silica nanocomposites

measured by fluorescence. European Polymer

Journal, 62, pp. 31-42.

DOI: 10.1016/j.eurpolymj.2014.10.018

(C) Elsevier, 2014

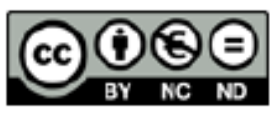

This work is licensed under a Creative Commons Attribution-NonCommercialNoDerivatives 4.0 International License. 


\title{
Interfacial characterization of epoxy/silica nanocomposites measured by fluorescence
}

\author{
Claire Antonelli a , Berna Serrano ${ }^{\mathrm{a}}$, Juan Baselga a , Rahmi Ozisik ${ }^{\mathrm{b}}$, Juan Carlos Cabanelas ${ }^{\mathrm{a}, *}$ \\ a Department of Materials Science and Engineering and Chemical Engineering, Universidad Carlos III de Madrid, Avda. Universidad, 30, 28911 Leganés, Madrid, \\ Spain \\ ${ }^{\mathrm{b}}$ Department of Materials Science and Engineering, and Rensselaer Nanotechnology Center, Rensselaer Polytechnic Institute, Troy, NY 12180, USA \\ * Corresponding author.E-mail address: caba@ing.uc3m.es (J.C. Cabanelas).
}

\begin{abstract}
Fluorescence labeling was used as a tool for the interfacial characterization of nanocom-posites. The solvatochromic probe dansyl chloride was employed as interfacial reporter in epoxy/silica nanocomposites. Molecular spacers (organosiloxanes and polyetheramines) of different lengths were used to vary the location of the chromophore at the interface. The steady state and time resolved fluorescent responses reflect a rigid polar interface. Fluores-cence changes during heating at a constant rate were analyzed for determining the local glass transition $(T g$ ) at the interface region. The fluorescence results were then compared to the $T g$ obtained from differential scanning calorimetry and the results showed the existence of a gradient interface of a few nanometers thick having different properties than the bulk matrix. The thickness of this interface is small but its altered dynamics due to strong interactions with the nanofiller spreads its influence throughout the whole matrix.

KEYWORDS

Interface

Polymer nanocomposites

Epoxy

Fluorescence

Glass
\end{abstract}

\section{Introduction}

The properties of polymer nanocomposites are associated with the dispersion and distribution state of the nanoparticles, and their interaction with the matrix. Their nanoscale size provide them with some of their most valued properties: low percolation threshold and high interfacial volume, which provides matrix-nanofiller interactions with different properties than the bulk polymer [14]. As a consequence, the overall properties of the combined system are altered compared to those of the traditional composite materials. One of the aspects that is still controversial is the comprehension of the interfacial architecture because of the complex sum of factors that determine its properties: physico-chemical interactions, topological effects and mechanical stresses among others. Specifically, the effects of the strength and the extent of the region of influence of the nanofillers on the polymer matrix are still not clear. There are even conflicting views on the definition of the extent of the interface. It is widely accepted that the properties of the interfacial region spread across the whole matrix affecting to a greater or lesser degree the structural relaxation dynamics of the polymer chains. The effect on the glass transition temper-ature $\left(T_{g}\right)$ is particularly of great importance, because it gives an idea on how polymer dynamics varies, and in addition, it is a key property for many applications. It is generally assumed that the $T_{g}$ tends to increase when favorable interactions exist between the nanofiller and the matrix. On the contrary, the presence of unfavorable interactions depresses the $T_{g}$. The extent of $T_{g}$ variation is also closely linked to the dispersion state the nanofiller $[5,6]$ because it defines the distribution and connectivity of the interfacial volume within the whole matrix and, as a consequence, the restriction level of chains mobility.

This behavior has been often analyzed in the literature on nanoconfinement effects on ultra-thin films. A $T_{g}$ 
depression is usually observed in free-standing ultra-thin films as the thickness of the film decreases [7]. This depression depends on the polymer-substrate interaction in supported thin films [8] and has led some authors to elaborate analogies between nanoconfinement and $T_{g}$ behavior in nanocomposites $[9,10]$. This apparent similarity has been used to justify some results in nanocomposites [11] even persisting some reluctance about this analogy [12,13]. In any case, it is generally postulated the existence of a polymeric layer around the nanoparticles whose properties are different from those of the bulk to explain the global behavior of the $T_{g}$ [13-17]. Filler-matrix or substrate-film attractive interactions reduce the local mobility of the polymeric chains $[8,13,16,17]$, whilst unfavorable interaction enhance the mobility [11]. For nanocomposites, the thickness of this layer is generally estimated between 1 and $10 \mathrm{~nm}$ depending on the studied system and on the employed experimental technique [18-22]. An important question that still remains open is to know in which way this polymer layer is able to impact the macroscopic properties of the nanocomposites. There are recent publications about this subject on thermoplastics filled with silica nanoparticles [13,22-25] but there is a lack of literature focused on thermosetting nanocomposites despite the important number of applications of these materials. Thermosetting nanocomposites are in fact complex systems due to the importance of the crosslinking density as it has been underlined by Brinson and coworkers [26]. In these materials the different $T_{g}$ behavior observed with the nanofiller content or the nanofiller-matrix interaction is often explained by the creation of heterogeneities in the network [27-29]. Anyways it is usual to observe an increase of the $T_{g}$ values in case of favorable interactions [30,31] and these obviously depend on the curing agent

[32] and on the nanofiller concentration [28,33].

Therefore, for a given system and its associated network structure, an important feature of nanocomposites is to know how the specific dynamics of the interface is propagated through the whole matrix. The object of this work is to improve the knowledge about how and to which extent the interfacial structure affects the whole thermosetting matrix. With this purpose, a fluorescent reporter will be placed on one end of several molecular spacers of varying size tethering both the probe and the nanofiller. The fluorescence of molecular probes is in fact an appreciated technique to study polymer materials [34]. It can provide information on a scale smaller than conventional light scattering and comparable to SANS [35]. For sensing molecular dynamics in condensed phases, a fluorescent dye should change its fluorescence signal on changes in the rotational diffusion of its surrounding media. It is well known the sensibility of some flurophores to rigidity, polarity or free volume variations of their close environment. Spectral shifts are caused by a combination of fluorophore-solvent interactions and charge transfer separation in the excited state but there is no single theory which account for all these effects [36,37]. Specifically, the so-called solvatochromic probes have large variations in their emission spectrum depending on the degree of stabilization of the excited state by the environment of the chromophore [38]. Since the pioneering work of
Loutfy [39] which related the variation of free volume of a PMMA matrix with the emission of a molecular rotor, fluorescence spectroscopy has been widely used to study thermal transitions in polymers. Torkelson and cowokers have extensively employed this technique to study confinement and relaxation processes in polymer films and nanocomposites [10,40-43]. Other authors have analyzed also thermal transitions in a high variety of polymer matrices [44-47].

The fluorescent marker selected for this work is 5(dimethylamino)naphthalene-1-sulfonyl chloride (Dansyl chloride, DNS), a well-known solvatochromic probe with charge transfer (CT) character. Intramolecular charge transfer in dansyl comes from the mixing of the $\pi-\pi^{*}$ transition of the aromatic ring and the lone electron pair of the amine $N\left(1-\pi^{*}\right)$ which acts as a donor [48]. This coupling is more efficient for the $1 \mathrm{~L}_{\mathrm{a}}$ (polar) state, and is the origin of a CT state. The $1 \mathrm{~L}_{b}$ (non-polar) state has less charge transfer capacity and remains in practice as a $\pi-\pi^{*}$ transition and it is usually called the locally excited (LE) state. Due to this charge transfer, excitation enhances very much more the dipole moment of the $1 \mathrm{~L}_{\mathrm{a}}$ state. Thus, in polar solvents, the emission comes from a CT state and is usually reflected by a red shift. On the contrary, in hydrophobic or non-polar solvents, the emission is mainly from the LE state. If the energy of both states is similar, the emission would come from both. In fact, DNS has been extensively used for solvent polarity studies, gelation and swelling or solvation dynamics in micellar environments [49-53]. Dansyl has been also employed to monitor microheterogeneity in surfaces [54] and polymer networks [55], curing behavior of thermosets [56,57], and thermal transitions and conformational changes in polymers, hybrid materials and composites [58,59]. In previous works, we used this fluorophore to study phase separation and morphology in blends [60] and the interface in PMMA nanocomposites [47].

In this work we have selected a low curing temperature system (DGEBA/m-xylylenediamine) as a model thermosetting system. As nanofiller we have selected silica nanoparticles that will be surface treated with functionalized silanes (short tethers) and with two Jeffamines (long tethers) of different size. DNS will be placed at the end of the tethers to probe different zones of the interfacial region. In addition, labeling of the hardener will allow characterizing the chromophore response when it is located in the bulk resin in presence of untreated silica nanoparticles. Along with the steady-state fluorescence, fluorescence decays will also be analyzed due to their higher sensitivity to the presence of transient states and relaxation phenomena. Finally, interfacial information will be confronted with macroscopic $T_{g}$ determined by calorimetry.

\section{Experimental section}

\subsection{Materials}

Colloidal suspensions of silica nanoparticles (DMAC-ST, $20 \%$ by weight in N,N-dimethylacetamide) were supplied by Nissan Chemical Industries, Ltd. Size distribution of 
the as received nanoparticles was determined by transmission electron microscopy (TEM) (Fig. S1 in Supplementary Information) to have an average value of $13.3 \pm 5.1 \mathrm{~nm}$. The specific surface area was evaluated by BET and was found to have an average value of $193 \pm 1 \mathrm{~m}^{2} / \mathrm{g}$. Both the average size and specific surface area agree with the values reported in literature [61].

The epoxy used in the current study as the matrix polymer was based on glycidyl-end capped poly(bisphenol A-co-epichlorohydrin) resin (DGEBA, $\quad M=348 \mathrm{~g} / \mathrm{mol}$, Sigma Aldrich) cured with $m$-xylylenediamine (MXDA, $M=136.19 \mathrm{~g} / \mathrm{mol}, 99 \%$, Sigma Aldrich).

Fluorescent probes were either mixed with the matrix or were attached to silica nanoparticle surfaces to probe the matrix or the interfacial region dynamics, respectively. The following fluorescent probes were used as received: dansyl chloride (DNS, 99\%, Invitrogen) and dansyl derivative $\mathrm{N}$ (dimethyl amino naphthalene sulfonyl)-1,5pentanediamine, which is usually known as dansyl cadaverine (DNSC, 99\%, Fluka). These fluorescent probes were attached to silica nanoparticles via various silane coupling agents: (3-Aminopropyl)dimethyl-ethoxysilane (APMES, 97\%, ABCR GMBH), (3-glicidoxypropyl)dimethyl-etoxysilane (GPMES, 97\%, ABCR GMBH), and N-octhyldimethylchlorosilane (ODMCS, 97\%, ABCR GMBH), which are designated as AS, GS and OS, respectively, to simplify nomenclature in the text. In addition, polyetheramines based on propyleneglycol (Jeffamine D230, $M=230 \mathrm{~g} / \mathrm{mol}$ and Jeffamine D400, $M=400 \mathrm{~g} / \mathrm{mol}$; Fluka) were used as spacers between silane coupling agents and fluorescent probes to be able to probe the silica/epoxy interface at different locations and as a function of distance from the silica nanoparticle surface. All solvents used in this work were GPC grade or superior. All concentrations are based on weight unless stated otherwise.

\subsection{Sample preparation and characterization}

\subsubsection{Silica surface modification}

Surface modification of silica nanoparticles with the monofunctional organosiloxanes, AS or GS, was performed following the same procedure in both cases. First, the silane coupling agent was reacted with the appropriate fluorescent probe (DNS for AS and DNSC for GS) in a $10 \%$ dilute DMAC solution at a fluorescent probe to silane molar ratio of $1: 40$. The reaction was carried out at $40{ }^{\circ} \mathrm{C}$ under $\mathrm{N}_{2}$ atmosphere until completion. The reaction of the probe with the silane was followed by thin layer chromatography. Next, the solution of the labeled silane was added dropwise to a silica nanoparticle suspension, which was previously diluted to $10 \%$ in DMAC, at room temperature and was stirred for $90 \mathrm{~min}$. The stability of the suspension was checked by dynamic light scattering (DLS, Malvern Zetasizer). Stoichiometric amounts of labeled silanes were used for condensation with the surface hydroxyl groups of silica nanoparticles. Stoichiometry was determined from specific surface area measurements and assuming 5 hydroxyl groups per unit area $\left(\mathrm{OH} / \mathrm{nm}^{2}\right)$ reactive sites on the nanoparticle [62]. Silica nanoparticle treatment with hydrophobic OS was performed following the same procedure used for AS and GS functionalization, however, because OS cannot be reacted with a fluorescent probe, a small amount of AS-DNS (with a AS:DNS molar ratio of $1.5: 1$ ) was grafted to the silica surface prior to silanization with OS. The molar ratio of OS:AS was 26:1 leading to a molar ratio of fluorescent probe to silane molar ratio of approximately $1: 40$.

In order to place the fluorescent probes at increasing distances away from the silica nanoparticle surface, Jeffamine chains were used as long spacers. First, the Jeffamine, D230 or D400 was labeled with DNS following the same reaction protocol that was used for silane coupling agents and described previously, however, the fluorescent probe to Jeffamine molar ratio was 1:4. Next, the DMAC/Jeffamine-DNS solution was added to a GStreated silica suspension in DMAC at a Jeffamine to GS molar ratio of 1:10 in order to achieve a similar fluorescent probe surface concentration in all samples. The structures of all chemical modifiers and the modi-fier:DNS molar ratios are presented in Table 1.

The presence of silane on nanoparticles was confirmed by FTIR (Perkin Elmer DX2000) by observing the absorption bands corresponding to the specific silane used or Jeffamine aliphatic backbones as described elsewhere [47]. The extension of surface modification (tethering density) was quantified by thermogravimetric analysis (TGA, Perkin Elmer TGA7) following the procedure described in Supplementary Information (Fig. S2).

\subsubsection{Nanocomposite preparation and characterization}

DMAC suspensions of modified silica were filtered (0.45 $\mu \mathrm{m})$ and added dropwise to a DMAC solution con-taining 10\% DGEBA under strong mechanical stirring that was maintained for $2 \mathrm{~h}$. The amount of added silica was monitored in order to achieve $2 \%$ nanoparticle concentration in the final nanocomposite. After mixing, samples were put in a high vacuum line at $70{ }^{\circ} \mathrm{C}$ for $90 \mathrm{~h}$ to com-pletely remove the solvent. To check the presence of unre-acted modifier in the final nanocomposite mixture, silica/DGEBA suspensions were dissolved in DMAC, centrifuged, and the supernatant solvent was analyzed by GPC. This procedure did not show any unreacted modifiers in the final nanocomposite mixtures. The modified silica/DGEBA mixtures were cured with the stoichiometric amount of MXDA at $80^{\circ} \mathrm{C}$ for $90 \mathrm{~min}$ and were post-cured at $130^{\circ} \mathrm{C}$ for $30 \mathrm{~min}$ to achieve completion of the reaction.

Some experiments used MXDA labeled with DNS for locating the probe in the matrix fixed to the polymer network, both for neat epoxy and nanocomposites prepared with bare (unmodified) silica. The labeling was done by reacting an adequate amount of DNS with the hardener at $40{ }^{\circ} \mathrm{C}$ under $\mathrm{N}_{2}$ atmosphere to obtain a concentration of $10^{-4} \mathrm{~mol} / \mathrm{kg}$ in the cured resin.

The main silica surface structures are qualitatively illustrated in Fig. 1. Surface modification with the two selected functional silanes (N2AD and N2GDC nanocomposites), lead to chemical functionalities that are able to react with the matrix (primary amino groups and oxirane rings). In these, the fluorophore is located close to the silica nanoparticle surface, especially in the case of AS treatment. In order to place the fluorophores further away from the silica nanoparticle surface, two different Jeffamines were used (indicated as N2D230 and N2D400 in Fig. 1). A low polarity 
Table 1

Structure of silica chemical modifiers, showing the tether: dansyl molar ratios and tether lengths.

\begin{tabular}{|c|c|c|c|}
\hline Modifier structure & Molar ratio & Tether length ${ }^{\mathrm{a}}$ & Designation \\
\hline & $40: 1$ & 5 & AS-DNS \\
\hline & $40: 1$ & 15 & GS-DNSC \\
\hline & $40: 10: 1$ & 21 & GS-D230-DNS $(n=2,5)$ \\
\hline & $40: 10: 1$ & 35 & GS-D400-DNS $(n=6,1)$ \\
\hline & $40:(1.5: 1)^{b}$ & & OS \\
\hline
\end{tabular}

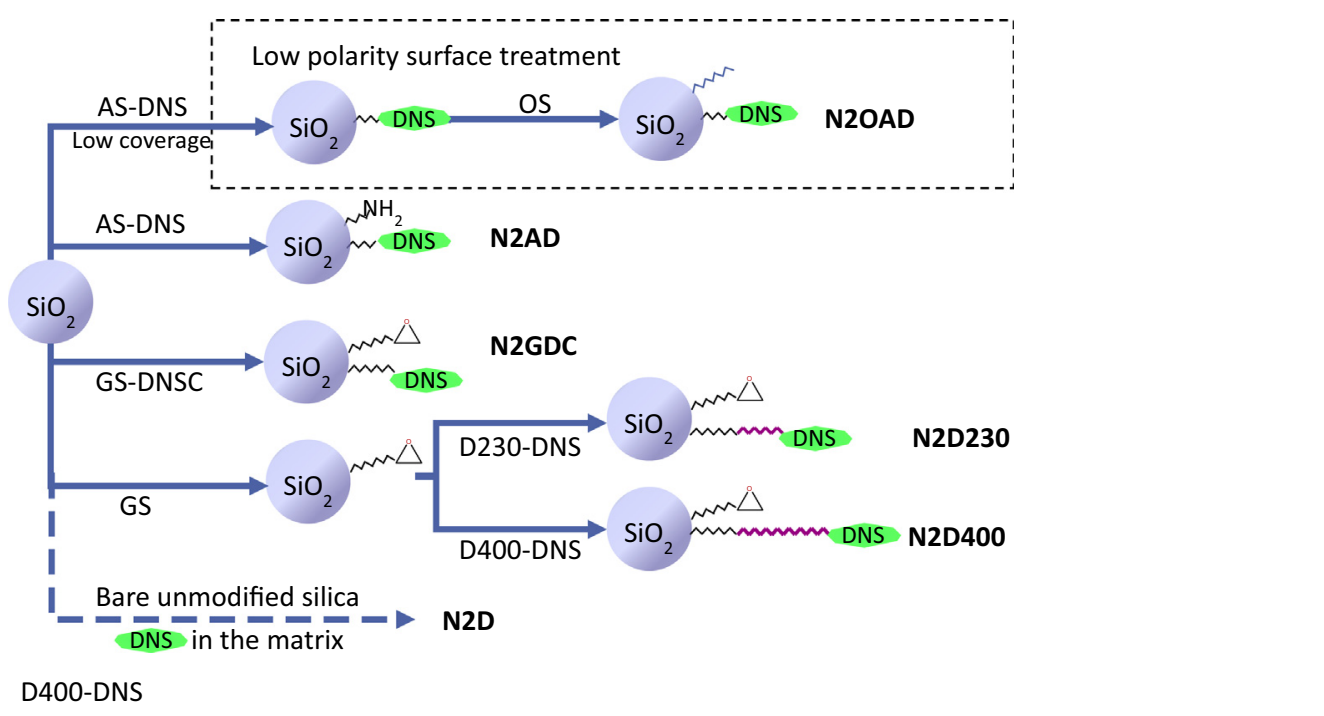

Fig. 1. Structures created as a result of surface modification of silica nanoparticles. The corresponding nanocomposite designations are indicated in bold.

reference for fluorescence studies was prepared by treating previously labeled nanoparticles with OS (N2OAD in Fig. 1). Finally, a nanocomposite was prepared with bare silica with the dansyl reporter located in the polymer matrix through MXDA (N2D nanocomposites).

It should be noticed for sample N2GDC that a possible addition of the secondary hydrogen of dansyl cadaverine to other glycydoxy moiety may take place. But this side reaction does not alter the length of the tether and has little effect on the average distance of the probe to the silica surface.

Glass transition temperatures $\left(T_{g}\right)$ of neat epoxy and silica/epoxy nanocomposites, defined at the mid-point of
$\Delta C_{P}$, were determined via differential scanning calorimetry (Perkin Elmer Pyris Diamond DSC). Scanning was performed under constant nitrogen gas flow using a sample mass of around $15 \mathrm{mg}$. The $T_{g}$ was evaluated from the second heating scan with a heating rate of $10^{\circ} \mathrm{C} / \mathrm{min}$ after cooling from $160{ }^{\circ} \mathrm{C}$ at the same rate.

Field emission scanning electron microscopy (SEM, Nova NanoSEM 230, FEI) was used to observe the morphology of fractured surfaces and the nanoparticle distribution in the nanocomposites. The samples were fractured after immersion in liquid nitrogen. A vibrational circular dichroism (VCD) detector was used applying an accelerating voltage between 4 and $7 \mathrm{kV}$. 


\subsubsection{Fluorescence measurements}

Fluorescence steady state measurements of DNSlabeled nanoparticles, embedded in the fully cured polymer matrix, were performed using an Edinburgh FS900 CDT instrument in the front face excitation mode. The excitation wavelength $\left(\lambda_{\text {exc }}\right)$ was set at $350 \mathrm{~nm}$ and emission scans were collected between 360 and $670 \mathrm{~nm}$ at $1 \mathrm{~nm}$ steps with $2 \mathrm{~mm}$ excitation and emission slits. For temperature dependent measurements, samples were put into aluminum pans $(15 \mathrm{mg}$ ) with a quartz cover into a Perkin Elmer DSC7 calorimeter. A bifurcated optical light guide coupled to the DSC, as described elsewhere [63], was used for excitation and emission collection. After heating the sample up to $160{ }^{\circ} \mathrm{C}$ and cooling it to $50^{\circ} \mathrm{C}$, temperature was increased in predetermined steps with adequate duration at each step to allow sample equilibration before spectra collection.

Analyses of the stationary spectra were performed by calculating the wavelength at the band maximum $\left(\lambda_{\max }\right)$, the full width at half maximum (FWHM), and the first moment of the emission spectra $(\langle v\rangle)$, which is defined as follows [36]:

$$
\langle v\rangle=\frac{\sum_{i} I_{F}\left(v_{i}\right) \cdot v_{i}}{I_{F}\left(v_{i}\right)}
$$

where $I_{F}\left(v_{i}\right)=I_{F}\left(\lambda_{i}\right) \cdot \lambda_{i}^{2}$. The first moment is related to the mean energy of emitted photons, has the advantage of having less fluctuations than fluorescence intensity, and is more sensitive to changes in the chromophore environment [64].

Fluorescence decays were measured using a pulse source (PDL 800-B, PicoQuant $\mathrm{GmbH}$ ) equipped with a $341 \mathrm{~nm}$ LED diode (PLS-340, PicoQuant GmbH, HBW of $14 \mathrm{~nm}$ ) with a sample chamber and an emission monochromator (Acton SP-150) coupled to a photomultiplier (PMA-M, Picoquant GmbH). Typical measurements were done at $5 \mathrm{MHz}, 78 \mathrm{ps} /$ channel and 20,000 counts at the maximum. Decays were obtained at emission wavelengths between 420 and $520 \mathrm{~nm}$ at $20 \mathrm{~nm}$ intervals. Deconvolution and fluorescence lifetime evaluation was performed with the PicoQuant Fluofit software. To check the accuracy of the equipment, the lifetimes of anthracene and 9, 10-diphenylanthracene in cyclohexane solution were measured, giving single exponential fits with values of 5.3 and $7.8 \mathrm{~ns}$, respectively, which are in very good agreement with literature [65].

\section{Results and discussion}

\subsection{Characterization of silica surface modification and nanocomposites}

The surface coverages (tethering densities) achieved for different treatments including the percent coverage, which was calculated on the basis of a silanol concentration of $5 \mathrm{OH} / \mathrm{nm}^{2}$ on bare silica [62], are provided in Table 2 . Results show that AS treatment achieves a better coverage (about 1.7 molecules $/ \mathrm{nm}^{2}$, which represents a mean silanol functionalization of 35\%) than GS and GS-Jeffamine surface treatments (about 1.3-1.4 molecules $/ \mathrm{nm}^{2}, 26-28 \%$ silanol
Table 2

The extent of silica surface modification as calculated from TGA curves.

\begin{tabular}{lll} 
Surface modifier & Molecules $^{\mathrm{a}}\left(\mathrm{nm}^{2}\right)$ & $\%^{\mathrm{b}}$ \\
\hline AS-DNS & $1.74 \pm 0.26$ & $35 \pm 5$ \\
GS-DNSC & $1.33 \pm 0.21$ & $26 \pm 4$ \\
GS-D230-DNS & $1.37 \pm 0.16$ & $27 \pm 3$ \\
GS-D400-DNS & $1.41 \pm 0.06$ & $28 \pm 1$ \\
OS + (AS-DNS) & $2.24 \pm 0.10$ & $45 \pm 2$ \\
\hline
\end{tabular}

a Molecules of tethered silane.

b Percent coverage was calculated from the average of three reactions.

coverage), in agreement with the greater reactivity of the amino silane towards siloxane condensation and the well-known autocatalytic effect of the amine [66]. The calculated tethering densities are comparable to values found in the literature for alkoxysilane grafted to silica in organic solvents $[67,68]$. The enhanced coverage reached by OS silanization is due to the greater reactivity of chlorosilanes compared to alkoxysilanes. In any case, the possibility of introducing off-stoichiometric epoxy:amine ratios when silica is functionalized with amine or epoxy groups may be discarded, as the total amount on the silica surface is negligible compared to the epoxy and amine concentrations in the bulk.

The glass transition temperatures of the fully cured nanocomposites obtained from DSC experiments are presented in Fig. 2. The glass transition temperatures of nanocomposites prepared without DNS were found to be similar to those containing DNS (not shown) confirming that DNS does not affect $T_{g}$ at the concentrations used in the current work. As can be seen in Fig. 2, the effect of the presence of silica nanoparticles on $T_{g}$ varied among different surface treatments. The $T_{g}$ increases moderately with respect to neat epoxy for all nanocomposites except for N2OAD.

In thermoset networks, it has been pointed out that the presence of nanofillers may disrupt the polymer network and also create non-stoichiometric regions, due to preferential adsorption of one of the components, therefore, decreasing $T_{g}$, although this effect is usually only important

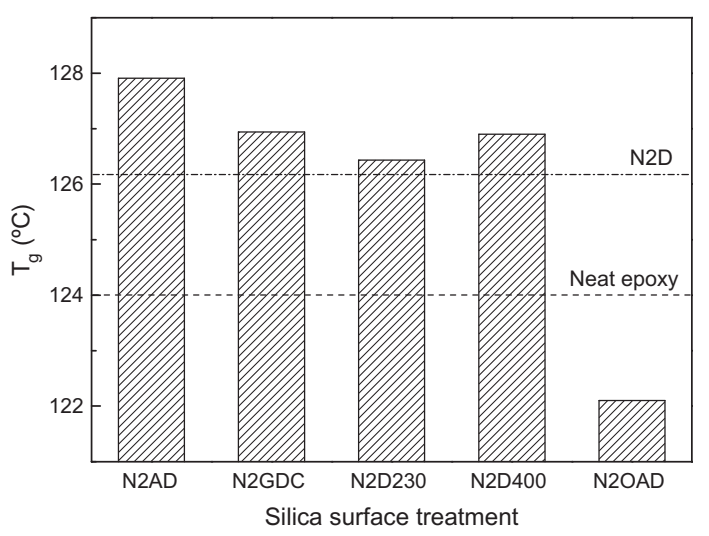

Fig. 2. The glass transition temperatures of neat epoxy and silica/epoxy nanocomposites as a function of silica surface treatment as obtained from DSC experiments. Horizontal dotted line is the $T_{g}$ of neat epoxy. 
at high concentrations $[27,69]$. On the other hand, an increase in $T_{g}$ may be observed when strong interactions between matrix and nanoparticles are present [70]; however, network disruption can partly compensate the restricted mobility caused by such strong interactions [26]. Taking this into account, it can be concluded that the systems investigated in the current study are characterized by strong interactions between either the surface silanol groups or the polar modifier groups and the epoxy matrix, with the exception of OS silanized sample (N2OAD), in which a more hydrophobic surface generated by the non-reactive silane causes a depression in $T_{g}$. Other than the OS silanization, all other surface modifications introduce functional groups that are able to covalently bond to the thermoset matrix, which may limit network disruption.

Selected SEM images of fractured surfaces are presented in Supplementary Information (Fig. S3). The presence of open clusters suggests some aggregation of the nanofillers, although energy dispersive X-ray analysis using the SiK line reveals the ubiquity of silica. The absence of holes caused by isolated silica particles or clusters detached from the fractured surfaces reflects a cohesive interface between the nanofillers and the epoxy matrix. Comparison of nanocomposites containing surface modified silica with those containing unmodified (bare) silica showed that short silane modifiers induce better dispersion in the samples studied.

\subsection{Steady state fluorescence of DNS-labeled nanocomposites}

The fluorescence spectra of free dansyl and silanedansylamide adducts in DMAC solution are presented in Supporting Information (Fig. S4). The emission maximum $\left(\lambda_{\max }\right)$ for all dansyl adducts appears at about $530 \mathrm{~nm}$ reflecting the high polarity of the DMAC solvent in agreement with published results $[48,51,71]$.

Emission spectra of DNS in the nanocomposites are presented in Fig. 3. A typical spectrum consists of a broad emission band that is blue shifted $50 \mathrm{~nm}$ with respect to DMAC solution in the 375-650 nm range. However, small differences can be appreciated for different systems as

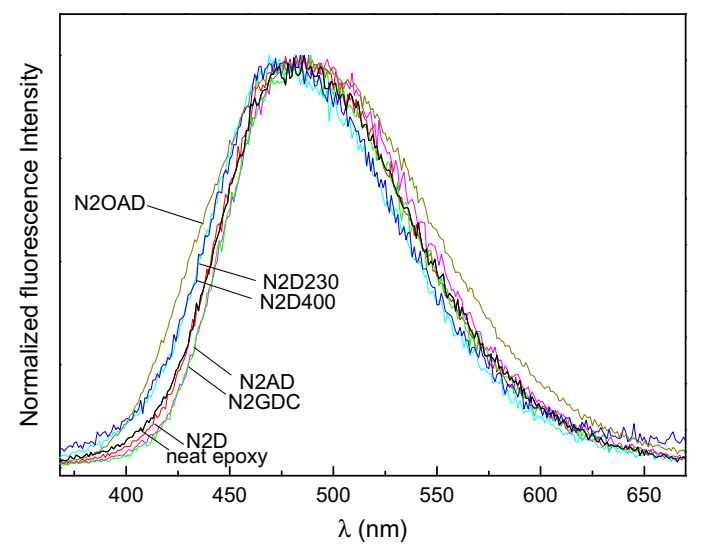

Fig. 3. Normalized fluorescence emission spectra of dansyl in neat epoxy and nanocomposites. reflected by the slight but significant changes in $\lambda_{\max }$ and FWHM. For each surface modification, the values of $\lambda_{\max }$ were obtained and plotted in Fig. 4 as function of tether length, which is expressed as the number of covalent bonds between silica and the chromophore. $\lambda_{\max }$ values are in a relatively narrow range of $478-487 \mathrm{~nm}$, which is characteristic of DNS emission in labeled polar surfaces [72,73]. A similar narrow range is also found for FWHM values (104-107 nm) except for the OS modified silica, in which FWHM is clearly greater $(\sim 127 \mathrm{~nm})$. The similarity in $\lambda_{\max }$ and FWHM along with the absence of shoulders or multiple emission bands suggest that in each sample most of the chromophores are in a common environment, i.e., labeled silica is completely embedded in the cured epoxy network providing DNS with a similar polar environment in all samples. For OS treatment (N2OAD nanocomposite), DNS emission comes from a wider distribution of population sites, a direct consequence of the dual silane modification, which generates different environments for the probe.

Emission shifts in charge transfer (CT) probes such as DNS are a result of two factors: polarity and rigidity of the environment. As the polarity of the probe environment increases, coupling efficiency between the excited state and the surrounding dipoles increases, and therefore, the probe emits from a more relaxed state and gives rise to a red shift. On the other hand, in rigid media, the dipolar relaxation of the probe microenvironment is slowed down so that it could not be completed during the excited state fluorescence lifetime. Consequently, the emission would take place from a high-energy, less-relaxed state, which gives rise to a blue shift. The fluorescence of DNS in the nanocomposites always takes place from a higher energy excited state than their respective emission in DMAC solutions as reflected by the blue shift in $\lambda_{\max }$. This is a direct consequence of having a more rigid environment and occurs because the solvation sphere in the epoxy matrix poorly relaxes, and therefore, the probe emits from a less relaxed state.

Although the local environments for all the studied treatments are polar, comparison of $\lambda_{\max }$ values as a function of the tether length may shed light on how the silica

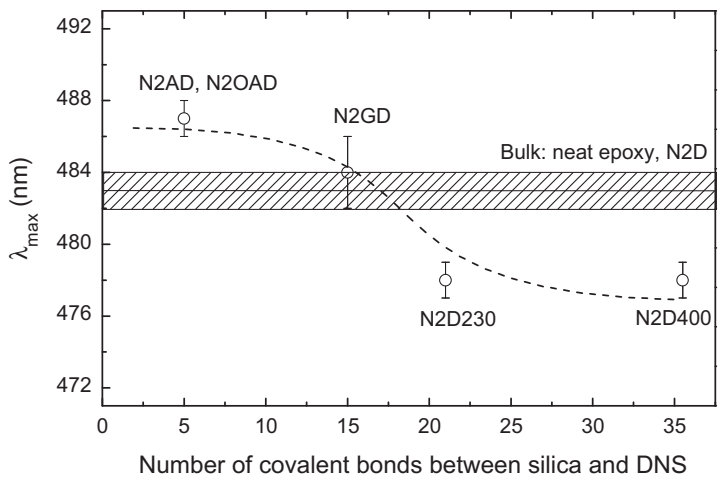

Fig. 4. Maximum wavelength of fluorescence emission spectra of DNS in silica/epoxy nanocomposites as a function of probe distance from silica nanoparticle surface. Dotted line is included as a guide to the eye. 
surface influences dansyl emission (see Fig. 4). It should be noted that the tether length used in Fig. 4 is the maximum distance between the silica surface and the probe, although dansyl will be located at the average distance resulting from all the possible tether conformations. As can be seen, a decrease in $\lambda_{\max }$ is observed as the tether length increases, which may be a consequence of progressively moving the probe from a hydroxyl-rich environment to a relatively less polar environment. Therefore, it is possible to state that dansyl is sensitive enough to the polarity changes that operate on the silica surface.

Fig. 4 provides an interesting comparison of the relative positions of $\lambda_{\max }$ when the probe is at the surface or dispersed in the polymer matrix. For probes dispersed in epoxy, $\lambda_{\max }$ is $483 \pm 1 \mathrm{~nm}$ whether there is silica or not in the matrix. The red-shifted emission of DNS attached to AS (N2AD and N2OAD) is attributed to the closer highly polar silica surface. The systems modified with Jeffamines are blue-shifted. This effect can be easily understood by considering the low polarity of the Jeffamine oxypropylene chains with respect to the DGEBA backbone, and the lack of segmental mobility due to the high crosslinking degree of the matrix in which the tethers are embedded.

\subsection{Fluorescence lifetimes}

DNS fluorescence lifetimes were measured for the nanocomposites. Fig. 5 shows the fluorescence decays obtained for N2AD at different emission wavelengths (at $20 \mathrm{~nm}$ intervals) as an example. Straight lines in the logarithmic plots prove that decays are essentially single exponentials, except at short wavelengths (at the blueedge of the emission spectra). However, two-exponential functions are required to obtain better fits to the data:

$i(t)=\sum_{i} A_{i} \exp \left(-t / \tau_{i}\right)$

where $t$ is the time after a pulse, $i(t)$ is the intensity of the emission at time $t$, and $A_{i}$ and $\tau_{i}$ are the amplitude and lifetime of the $i$ th exponential decay. Decay lifetimes are shown in Table 3 at $480 \mathrm{~nm}$ emission wavelength along with the normalized amplitude $A_{i}$ and relative intensity $f_{i}$

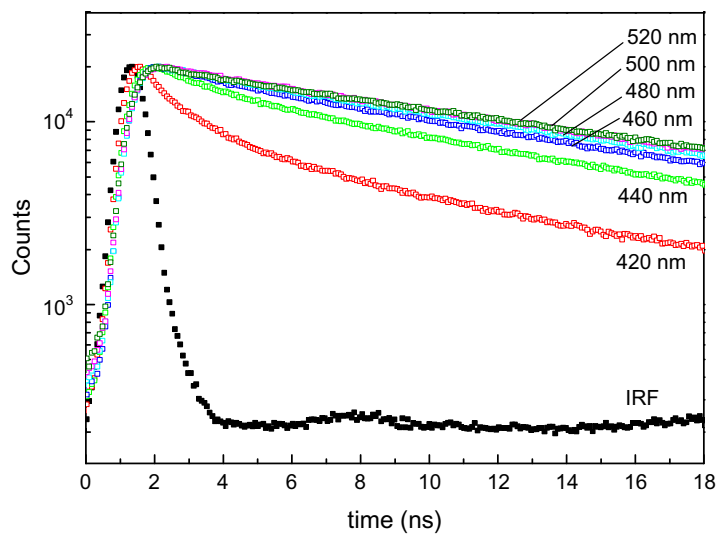

Fig. 5. Fluorescence decays of N2AD at different emission wavelengths. IRF is the instrument response function.
Table 3

Two-exponential fits of fluorescence decay at $480 \mathrm{~nm}$ emission wavelength.

\begin{tabular}{llllll}
\hline Sample & $\tau_{1}(\mathrm{~ns})$ & $\tau_{2}(\mathrm{~ns})$ & $\alpha_{1}{ }^{\mathrm{a}}$ & $f_{1}{ }^{\mathrm{b}}$ & $\chi^{2}$ \\
\hline Neat epoxy & $12.95 \pm 0.03$ & $1.62 \pm 0.04$ & 0.75 & 0.96 & 1.08 \\
N2D & $13.12 \pm 0.04$ & $1.75 \pm 0.03$ & 0.75 & 0.96 & 1.17 \\
N2AD & $13.91 \pm 0.04$ & $1.83 \pm 0.10$ & 0.87 & 0.98 & 1.19 \\
N2GDC & $14.37 \pm 0.04$ & $1.50 \pm 0.08$ & 0.86 & 0.98 & 1.27 \\
N2D230 & $12.83 \pm 0.04$ & $1.55 \pm 0.04$ & 0.72 & 0.96 & 1.18 \\
N2D400 & $12.80 \pm 0.04$ & $1.60 \pm 0.03$ & 0.71 & 0.95 & 1.14 \\
N2OAD & $13.78 \pm 0.04$ & $1.70 \pm 0.10$ & 0.92 & 0.99 & 1.11 \\
\hline
\end{tabular}

a Normalized amplitude.

b Relative intensity.

(i.e., the normalized contribution of each lifetime to the total intensity in the stationary spectrum), calculated according to the following equations [37]:

$\alpha_{i}=A_{i} / \sum_{j}^{\infty} A_{j}$

$f_{i}=\frac{\alpha_{i} \tau_{i}}{\sum_{j}^{\infty} \alpha_{j} \tau_{j}}$

Two-exponential functions give reasonably good fits with $\chi^{2}$ values below 1.3 in all decays but, as shown by the $f$ values (very close to 1 ), the main contribution to the stationary spectra comes from the long lifetime, which ranges between 12 and $14 \mathrm{~ns}$, in good agreement with data reported for dansyl in other polar and viscous media $[50,73]$, and its contribution increases with emission wavelength that will be presented in the following sections. The short lifetime is only important at lower wavelengths and has a constant value at around $1.7 \mathrm{~ns}$.Long lifetime, $\tau_{1}$, is plotted as a function of emission wavelength for all studied systems in Fig. 6 . It can be clearly observed that $\tau_{1}$ increases with emission wavelength. The relative intensity, $f_{1}$, values also increase with emission wavelength (see Fig. S5). It is worth noting that three samples (N2AD, $\mathrm{N} 2 \mathrm{GDC}$ and N2OAD) presented higher lifetimes than that of neat epoxy, N2D and nanocomposites with dansyl

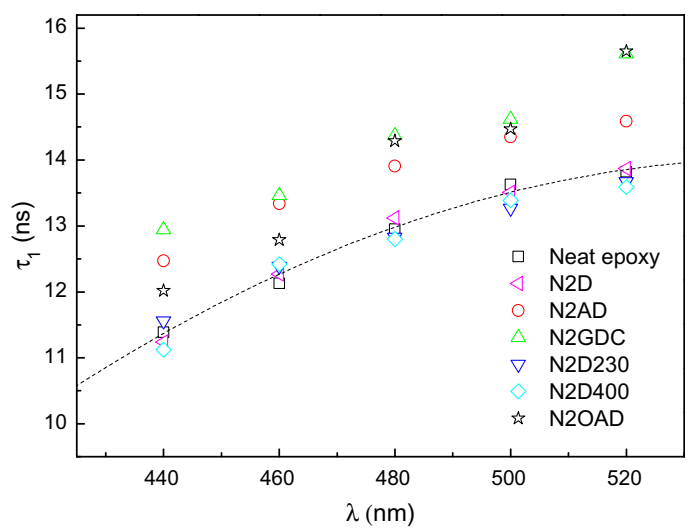

Fig. 6. Long lifetime, $\tau_{1}$, as a function of emission wavelength for dansyl in various samples. In neat epoxy and N2D, dansyl is linked to the epoxy network. In all other samples, dansyl is tethered to the silica nanoparticles. Dash line is included as a guide for the neat epoxy data. 
located through the Jeffamine moiety. These three samples share the common feature of having dansyl being very close to the silica surface and are the same ones that show a red shift in their stationary spectra (Fig. 4).

The physical meaning of multi-exponential decays is not usually straightforward. DNS decays in heterogeneous condensed phases can be attributed to many factors: the ability for internal rotation of the dimethylamino group, the efficiency of the non-radiative processes and the solvent relaxation dynamics; all of which depend on the polarity and viscosity of the microenvironment. In spite of this, the observation of a rise in the lifetime with increasing emission wavelength means that the long life-time corresponds to a high dipole moment charge transfer complex that is stabilized by the polar surroundings of the probe. The emission from relaxed states (long wavelengths) comes from molecules with a longer lifetime than the relaxation time of the solvent (the polymer matrix in the current work), and the high-energy emission (low wavelengths) comes from molecules that decay when the coupling with the solvent is not completed. Therefore, Fig. 6 reflects a relaxation phenomenon that appears in all samples and agrees with earlier observations of Ghigg-ino and co-workers [74]. There are not many published data about excited state relaxation dynamics in condensed phases. Charge-transfer (CT) reaction and the reorganization of solvent molecules in low viscosity polar media are very fast, on the order of ps [75]; in which case the solvent responds virtually immediately to the new electronic configuration of the probe. But when the CT reaction and solvent dynamics are of the same order of magnitude, the fluorescence monitors the environment relaxation. For example, Sarkar [53] used DNS to study the surface relaxation on DNS-labeled charged micelles and found a solvent relaxation time constant of 338 ps. A higher relaxation time, on the order of dansyl fluorescence lifetime, is to be expected for the more rigid systems studied in the current work. Finally, the short lifetime with very low contribution does not change with emission wavelength and its contribution decreases in the red side of the spectrum (Fig. S5) as it is not relaxed by the solvent, it may belong to emission from a low energy (LE) state that is not stabilized in polar media, as it was reported for DNS immobilized in chitosan [73].

The higher lifetimes obtained for N2GDC, N2AD and N2OAD samples reflect a more rigid environment that restricts non-radiative deactivation. Therefore, lifetime measurements confirm that high polarity and rigidity can explain steady state results for these samples.

\subsection{Temperature dependence of steady state fluorescence: determination of the local glass transition}

The fluorescence of the silica/epoxy nanocomposites was monitored as a function of temperature as described in the experimental section. As temperature was increased from 50 to $150{ }^{\circ} \mathrm{C}$, the fluorescence intensity decreased because of non-radiative processes, which is commonly observed in condensed polymer phases [40,76,77]. In addi-tion, a red shift is observed and the first moment of the emission spectra, $\langle v\rangle$, gradually decreased approximately $600 \mathrm{~cm}^{-1}$ as depicted in Fig. 7.

Temperature effects on fluorescence spectra are typically complex. On the one hand, an increase in temperature may modify the population level of both ground and excited vibronic states and may manifest as a red shift in the emission. Assuming a value of $\sim 1000 \mathrm{~cm}^{-1}$ for the energy difference between vibronic states [78], a tempera-ture increase of about $100 \mathrm{~K}$, as is the case in the current study, should reduce the population of the zero vibrational level of the ground state from $99 \%$ to $97 \%$; and the associ-ated red shift can be roughly estimated to be $40 \mathrm{~cm}^{-1}$, one order of magnitude lower than that experimentally observed. On the other, when solvent relaxation rate is much lower than excited lifetime, temperature weakens dipole-dipole interactions [79] destabilizing the excited probe-solvent coupling, and therefore, inducing a blue shift. Therefore, in conjunction with the lifetime results presented previously, the large red shifts observed for all samples must be related to solvent relaxation time effects. As temperature increases, the solvent relaxation becomes faster and the excited probesolvent coupling becomes progressively more efficient in stabilizing the excited state. This phenomenology was already observed for DNS in frozen solvents [74].

However, in condensed phases, other temperature dependent phenomena such as quenching or non-radiative processes may affect the quantum yield and the lifetime, thereby, making it extremely difficult to identify the effect of each factor on the fluorescence spectra of DNS and to predict its temperature dependence. The combination of all these possible factors might be the origin of the experimentally observed almost linear trend of $\langle v\rangle$ with temperature.

Inspection of Fig. 7 clearly shows a slope change in the average energy emission near the glass transition of the epoxy (see Fig. 2). The temperature at which this change appears is identified as $T_{\text {fluo }}$. Slope changes in fluorescence intensity with temperature are usually attributed to the onset of a polymer relaxation process $[40,42,46,47]$. In the current study, the decrease of $\langle v\rangle$ is more pronounced

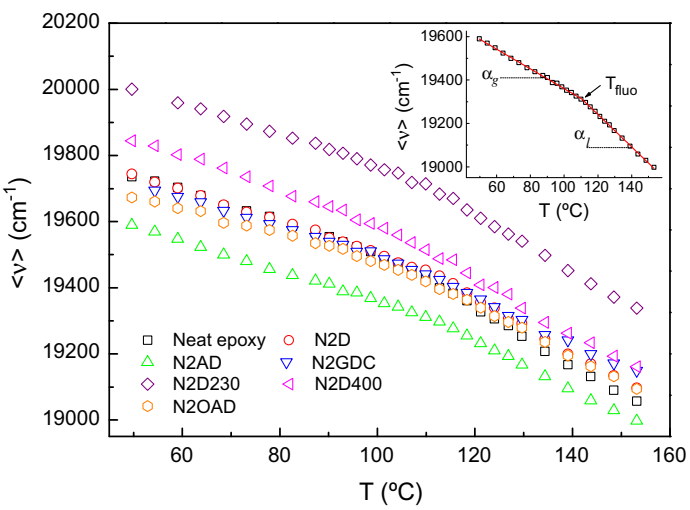

Fig. 7. Temperature dependence of the first moment of fluorescence emission, $\langle v\rangle$, for neat sample (bulk localization) and nanocomposites. Insert: Determination of $T_{\text {fluo }}$ from the variation of $\langle v\rangle$ with temperature for N2AD sample by a bilinear fit. 
in the temperature range corresponding to the elastomeric state, where matrix properties change more than those in the glassy state. Therefore, the $\langle v\rangle$ dependence on temperature follows the same trend as the properties of the matrix, i.e., smooth below the transition and more pronounced above it. To evaluate $T_{\text {fluo }}$, data were fitted to a continuous bilinear function according to the following expression, where $\alpha_{g}$ and $\alpha_{l}$ are the slopes in the glassy and elastomeric states, respectively:

$$
v(T)=v\left(T_{\text {fluo }}\right)+\frac{\alpha_{g}+\alpha_{l}}{2}\left(T-T_{\text {fluo }}\right)+\frac{\alpha_{l}-\alpha_{g}}{2}\left|T-T_{\text {fluo }}\right|
$$

This method has been successfully used previously to monitor the emission of a solvatochromic probe in a polymeric matrix during physical aging [80]. The mathematical function provided good fits to the experimental data. An example fitting to the N2AD data is presented in the insert of Fig. 7.

Table 4 summarizes the bilinear fitting parameters obtained for all samples: the slopes in the glassy, $\alpha_{g}$, and elastomeric state, $\alpha_{l} ; T_{\text {fluo }}$; and $\langle v\rangle\left(T_{\text {fluo }}\right) . \alpha_{g}$ values were found to be in the $4-5 \mathrm{~cm}^{-1}{ }^{\circ} \mathrm{C}^{-1}$ range, showed no trend, and were quite similar irrespective of the location of the DNS in the sample. In the elastomeric state, $\alpha_{l}$ values were found to be in the $8-9 \mathrm{~cm}^{-1}{ }^{\circ} \mathrm{C}^{-1}$ range for samples in which DNS was located in the bulk polymer or attached to the silica particles using long tethers. However, when DNS is located close to the silica surface (N2AD, N2OAD and N2GDC samples), the $\alpha_{l}$ values are clearly smaller reflecting a lower capability of the microenvironment for DNS excited state relaxation.

Concerning $T_{\text {fluo }}$ values, it is important to point out that there is no valuable reason for their coincidence with $T_{g}$ as they correspond to processes operating in very different time scales. It is common in the literature to find this match, using fluorescence intensity measurements, as a token of good quality of the measurement by the fluorescence technique [40]. However, the change of the fluorescence emission energy with temperature depends on the coupling between the excited state of the chromophore and the dipoles in the vicinity of the probe rather than on the long range cooperative motions responsible for the glass transition. Therefore, $T_{\text {fluo }}$ should appear before $T_{g}$ as it is experimentally observed.

In Fig. 8, the variation of $T_{\text {fluo }}$ is presented as function of spacer length, as expressed by the number of covalent Table 4

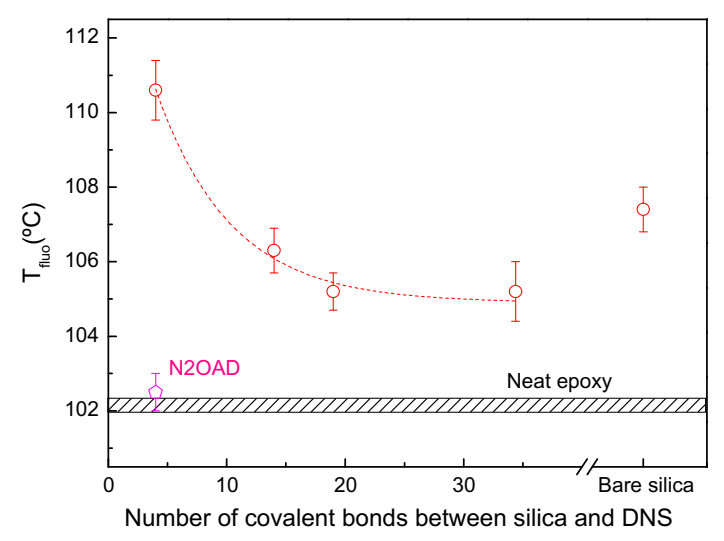

Fig. 8. $T_{\text {fluo }}$ as a function of silica-dansyl tether length. Straight band represents $T_{\text {fluo }}$ of neat epoxy. The values of nanocomposites with bare silica are presented on the right hand side of the plot for comparison purposes.

bonds, and the $T_{\text {fluo }}$ value for DNS in neat epoxy is presented as a horizontal band. Nanocomposites with DNS located close to the silica surface show the highest $T_{\text {fluo }}$ values suggesting a more rigid environment, which is in agreement with the higher lifetimes and smaller $\alpha_{l}$ values observed. When the tether length is increased, $T_{\text {fluo }}$ values approach those obtained when DNS is located in the bulk. This fact reflects the incremental probability of DNS being further away from the hydrophilic surface.

Data from Fig. 8 suggests an increasing trend in flexibility as the probe distance from the surface of the nanoparticles increases, which, in turn, suggests the presence of a flexibility gradient within the interfacial region, i.e., the matrix dynamics become progressively more restricted as the probe gets closer to the nanoparticle surface.

A convenient means to characterize a gradient consists of measuring the difference in transition temperatures between the interface and the bulk polymer. It is noteworthy that the differences observed in $T_{\text {fluo }}$ are higher than the differences observed in $T_{g}$. For example, in the sample $\mathrm{N} 2 \mathrm{AD}, T_{\text {fluo }}$ changes $+8{ }^{\circ} \mathrm{C}$ while $T_{g}$ changes only $+4{ }^{\circ} \mathrm{C}$. Since it is a macroscopic measurement, $T_{g}$ measures the dynamics of the interface/bulk ensemble. As mentioned before, $T_{g}$ variations in nanocomposites are usually attributed to changes in the rigidity of the interface but no experimental evidence is usually provided for such a statement. The

Bilinear fitting parameters obtained for the variation of $\langle v\rangle$ with temperature for neat epoxy and silica/epoxy nanocomposites.

\begin{tabular}{|c|c|c|c|c|}
\hline Sample & $\alpha_{g}\left(\mathrm{~cm}^{-1}{ }^{\circ} \mathrm{C}^{-1}\right)$ & $\alpha_{I}\left(\mathrm{~cm}^{-1}{ }^{\circ} \mathrm{C}^{-1}\right)$ & $T_{\text {fluo }}\left({ }^{\circ} \mathrm{C}\right)$ & $v\left(T_{\text {fluo }}\right)\left(\mathrm{cm}^{-1}\right)$ \\
\hline \multicolumn{5}{|c|}{ DNS in bulk polymer } \\
\hline Neat epoxy & $-4.6 \pm 0.1$ & $-8.8 \pm 0.1$ & $102.2 \pm 0.2$ & $19,496 \pm 3$ \\
\hline N2D & $-4.6 \pm 0.1$ & $-8.2 \pm 0.2$ & $107.4 \pm 0.6$ & $19,472 \pm 4$ \\
\hline \multicolumn{5}{|c|}{ DNS at the interface } \\
\hline $\mathrm{N} 2 \mathrm{AD}$ & $-4.5 \pm 0.1$ & $-7.4 \pm 0.1$ & $110.6 \pm 0.8$ & $19,311 \pm 4$ \\
\hline N2OAD & $-4.0 \pm 0.1$ & $-7.5 \pm 0.1$ & $102.5 \pm 0.5$ & $19,477 \pm 3$ \\
\hline N2GDC & $-4.4 \pm 0.1$ & $-7.2 \pm 0.2$ & $106.3 \pm 0.6$ & $19,468 \pm 4$ \\
\hline N2D230 & $-4.5 \pm 0.1$ & $-8.7 \pm 0.1$ & $105.2 \pm 0.5$ & $19,749 \pm 3$ \\
\hline N2D400 & $-5.1 \pm 0.1$ & $-9.3 \pm 0.4$ & $105.2 \pm 0.8$ & $19,567 \pm 6$ \\
\hline
\end{tabular}


results obtained in the current study clearly demonstrate that the interfacial interaction has a long-range effect that spreads throughout the whole matrix. In addition, the region of direct influence of the nanoparticles seems to vanish after 20-30 chemical bonds, which corresponds to an extension of less than approximately $3 \mathrm{~nm}$. This result is in agreement with previous studies that postulate the existence of a vitreous region of a few nanometers in length around the nanoparticle $[18,19]$.

Nanocomposite based on OS treated silica (N2OAD) is the only sample with unfavorable silica-matrix interactions due to the hydrophobic nature of the silane used. Modification by OS leads to decreased $T_{\text {fluo }}$ in the same way as observed in $T_{g}$ measurements. Therefore, strong or poor interactions translate into high or low $T_{\text {fluo }}$ values with respect to the neat epoxy. In addition, in sample N2OAD, where dansyl is located at the interface, $T_{\text {fluo }}$ is on the order of that observed for DNS in the neat epoxy in spite of the high polarity of the silica surface where DNS is located. This means that fluorescence is being able to detect changes in the interfacial interactions both in the bulk and in the interface.

In addition to the interactions within the interface, another antagonistic effect on the dynamics of highly crosslinked networks should be noted: network disruption due to the physical presence of nanoparticles, which may promote relaxation. This fact has been underlined by Brinson and coworkers in their work on epoxy/CNT nanocomposites [26], where particle-matrix interactions were weak. These authors found that network disruption due to the presence of the nanofillers decreased the $T_{g}$ only for systems in which crosslink density was high. To evalu-ate the effect of network disruption, we have determined the size of cooperative rearranging region (CRR) according to the method proposed by Donth [81], which is based on the thermal fluctuation theory and DSC measurements. In this approach, the characteristic length scale of heteroge-neity $(\xi)$ is calculated according to the following:

$\xi^{3}=\frac{k_{B} T_{g}^{2} \Delta\left(1 / c_{v}\right)}{\rho \delta T^{2}}$

where $k_{B}$ is the Boltzmann constant, $\Delta\left(1 / c_{v}\right)$ can be replaced by the heat capacity at constant pressure $\Delta\left(1 / c_{p}\right)$ obtained by DSC, $\rho$ is the density of the material, and $\delta T$ is the mean temperature fluctuation of one CRR of volume $\xi^{3}$. Details of the calculation method can be found elsewhere [81,82]. For the neat epoxy resin (with no nano-particles), the calculated CRR length is $2.2 \mathrm{~nm}$, which is in agreement with the values found in the literature for fully cured epoxy networks with comparable $T_{g}$ values $[26,83,84]$. In the case of nanocomposites, the CRR length increases slightly having values of $3.0 \pm 0.7 \mathrm{~nm}$, irrespec-tive of the surface treatment. We attribute this enhance-ment in $\xi$ to a balance of two opposing phenomena: network disruption induced by the nanoparticles and creation of an interface with restricted mobility, where the latter compensates the former. A similar result was found by Pistor et al. [84] working with epoxy/POSS nanocomposites, however, the $T_{g}$ of the nanocomposites was lower than that of neat epoxy.
Srivastava and Basu [85], working with gold/PMMA weak nanocomposites, proposed a model to understand the relation between the interfacial width, $\xi$, and the sign of $T_{g}$ deviation, which should be valid for confined polymers. According to these authors, when the interface width is shorter than $\xi$, the mean relaxation of the composite is dominated by the dynamics of the surface polymer layer. On the other hand, for interfacial regions larger than $\xi$, the interface dominates the relaxational behavior of the ensemble. However, it is not clear if this model could apply for thermosetting polymers since a large and diffuse interface can only be a consequence of an extended network disruption that would unavoidably lead to a decrease in $T_{g}$. In the current study, the $T_{g} s$ of the nanocomposites (with the exception of N2OAD) are greater than that for the neat resin. Therefore, although the width of the interface and $\xi$ are similar, it seems that the strong polymer-silica interaction affects the dynamics of the neighboring polymer segments, and as a result, spreading the effect throughout the whole matrix. More work is being carried out on the structural relaxation of chain segments and chain dynamics at the interface.

\section{Conclusions}

In the current study, the bulk and interfacial properties of silica/epoxy nanocomposites were examined by fluorescent probes that were either linked to the matrix network or were tethered to silica nanoparticles, thereby, placing them at the silica-epoxy interface. In addition, the fluorescent probe distance to silica surface was varied by using different silane coupling agents or silane coupling agents and chain extenders, enabling the study of interfacial dynamics and relaxation at the interface as a function of distance from the nanoparticle surface. A detailed analysis of the fluorescent response of the probe and its dependence on temperature allowed a molecular scale interpretation of the changes observed in the glass transition temperature of the silica/epoxy nanocomposites and its relation to the interfacial dynamics.

Steady state and time resolved measurements revealed that fluorophores located close to the silica nanoparticle are in a more rigid environment than those located in the polymer bulk. This may be a consequence of the strong interfacial interactions that reduce the ability of the probe to relax the excited state. Also, the glass transition temperature of the neat epoxy as determined by DSC increased with the addition of silica nanoparticles. These two observations, when coupled together, suggest a close relationship between the interfacial rigidity and the macroscopic glass transition temperature.

The first important conclusion of the current study is concerned with the confirmation of rigidity effects at the interface and its percolation throughout the matrix. An increase in the glass transition temperature is usually attributed to the existence of a rigid polymer layer around the particles; however, this statement probably requires more experimental evidence. Our results on silica/epoxy nanocomposites show that when the probe is located very close to the nanoparticle surface (such as in system N2AD), 
its stationary spectra reflects a rigid environment, which has been confirmed by an increased fluorescence lifetime. Accordingly, the analysis of the temperature dependence of the probes' average emission energy reveals that the local relaxation temperature $\left(T_{\text {fluo }}\right)$ at the interface is greater than that when the probe is located in the bulk. The concurrent measurement of the glass transition temperature yields a value greater than that of the neat epoxy; therefore, confirming that rigidity at the interface is translated throughout the whole sample.

The second important conclusion is related to the existence of a rigidity gradient and its extension into the bulk from the nanoparticle surface. By varying the tether length that connects the fluorescent probes to the nanoparticles, it was possible to measure a length scale for the region of direct influence of the nanoparticles. In the case of silica/epoxy systems studied, this length scale was found to be less than approximately $3 \mathrm{~nm}$. The fluorescence response of probes was found to differ from the fluorescence response of probes that were ramdonly linked to the matrix, and was found to strongly and inversely depend on the average probe-nanoparticle distance, thereby, proving the existence of an interface gradient.

\section{Acknowledgments}

J.C. Cabanelas, C. Antonelli, J. Baselga and B. Serrano gratefully acknowledge Spanish Ministerio de Educación, Cultura y Deporte (MAT2010-17091) for financial support. R. Ozisik acknowledges support from the United States National Science Foundation under Grant No. 1200270.

\section{References}

[1] Kango S, Kalia S, Celli A, Njuguna J, Habibi Y, Kumar R. Surface modification of inorganic nanoparticles for development of organicinorganic nanocomposites-A review. Prog Polym Sci 2013;38: 123261.

[2] Ash BJ, Siegel RW, Schadler LS. Mechanical behavior of alumina/poly (methyl methacrylate) nanocomposites. Macromolecules 2004;37: 1358-69.

[3] Flory AL, Ramanathan T, Brinson LC. Physical aging of single wall carbon nanotube polymer nanocomposites: effect of functionalization of the nanotube on the enthalpy relaxation. Macromolecules 2010;43:4247-52.

[4] Akcora P, Liu H, Kumar SK, Moll J, Li Y, Benicewicz BC, et al. Anisotropic self-assembly of spherical polymer-grafted nanoparticles. Nat Mater 2009;8:354-9.

[5] Qiao R, Deng H, Putz KW, Brinson LC. Effect of particle agglomeration and interphase on the glass transition temperature of polymer nanocomposites. J Polym Sci Part B Polym Phys 2011;49:740-8.

[6] Natarajan B, Li Y, Deng H, Brinson LC, Schadler LS. Effect of interfacial energetics on dispersion and glass transition temperature in polymer nanocomposites. Macromolecules 2013;46:2833-41.

[7] Keddie JL, Jones RAL, Cory RA. Size-dependent depression of the glass transition temperature in polymer films. Europhys Lett 1994;27: 5964.

[8] Keddie JL, Jones RAL, Cory RA. Interface and surface effects on the glass-transition temperature in thin polymer films. Faraday Discuss 1994;98:219-30.
[9] Bansal A, Yang H, Li C, Cho K, Benicewicz BC, Kumar SK, et al. Quantitative equivalence between polymer nanocomposites and thin polymer films. Nat Mater 2005;4:693-8.

[10] Rittigstein P, Priestley RD, Broadbelt LJ, Torkelson JM. Model polymer nanocomposites provide an understanding of confinement effects in real nanocomposites. Nat Mater 2007;6:278-82.

[11] Ash BJ, Schadler LS, Siegel RW. Glass transition behavior of alumina/ polymethylmethacrylate nanocomposites. Mater Lett 2002;55: 837.

[12] Pryamitsyn V, Ganesan V. A comparison of the dynamical relaxations in a model for glass transition in polymer nanocomposites and polymer thin films. Macromolecules 2010;43:5851-62.

[13] Harton SE, Kumar SK, Yang H, Koga T, Hicks $K$, Lee $H$, et al. Immobilized polymer layers on spherical nanoparticles. Macromolecules 2010;43:3415-21.

[14] Berriot J, Montes H, Lequeux F, Long D, Sotta P. Evidence for the shift of the glass transition near the particles in silica-filled elastomers. Macromolecules 2002;35:9756-62.

[15] Pye JE, Rohald KA, Baker EA, Roth CB. Physical aging in ultrathin polystyrene films: evidence of a gradient in dynamics at the free surface and its connection to the glass transition temperature reductions. Macromolecules 2010;43:8296-303.

[16] Fryer DS, Peters RD, Kim EJ, Tomaszewski JE, De Pablo JJ, Nealey PF, et al. Dependence of the glass transition temperature of polymer films on interfacial energy and thickness. Macromolecules 2001;34: 5627-34.

[17] Fryer DS, Nealey PF, De Pablo JJ. Thermal probe measurements of the glass transition temperature for ultrathin polymer films as a function of thickness. Macromolecules 2000;33:6439-47.

[18] Fragiadakis D, Bokobza L, Pissis P. Dynamics near the filler surface in natural rubber-silica nanocomposites. Polymer 2011;52:3175-82.

[19] Fragiadakis D, Pissis P, Bokobza L. Modified chain dynamics in poly. (dimethylsiloxane)/silica nanocomposites. J Non Cryst Solids 2006; 352:4969-72.

[20] Sargsyan A, Tonoyan A, Davtyan S, Schick C. The amount of immobilized polymer in PMMA $\mathrm{SiO}_{2}$ nanocomposites determined from calorimetric data. Eur Polym J 2007;43:3113-27.

[21] Arrighi V, McEwen I, Qian H, Serrano Prieto M. The glass transition and interfacial layer in styrene-butadiene rubber containing silica nanofiller. Polymer 2003;44:6259-66.

[22] Jouault N, Moll JF, Meng D, Windsor K, Ramcharan S, Kearney C, et al. Bound polymer layer in nanocomposites. ACS Macro Lett 2013;2: 371-4.

[23] Bogoslovov RB, Roland CM, Ellis AR, Randall AM, Robertson CG. Effect of silica nanoparticles on the local segmental dynamics in poly (vinyl acetate). Macromolecules 2008;41:1289-96.

[24] Moll J, Kumar SK. Glass transitions in highly attractive highly filled polymer nanocomposites. Macromolecules 2012;45:1131-5.

[25] Holt AP, Sangoro JR, Wang Y, Agapov AL, Sokolov AP. Chain and segmental dynamics of poly(2-vinylpyridine) nanocomposites. Macromolecules 2013;46:4168-73.

[26] Putz KW, Palmeri MJ, Cohn RB, Andrews R, Brinson LC. Effect of cross-link density on interphase creation in polymer nanocomposites. Macromolecules 2008;41:6752-6.

[27] Becker O, Varley R, Simon G. Morphology, thermal relaxations and mechanical properties of layered silicate nanocomposites based upon high-functionality epoxy resins. Polymer 2002;43:4365-73.

[28] Preghenella M, Pegoretti A, Migliaresi C. Thermo-mechanical characterization of fumed silica-epoxy nanocomposites. Polymer 2005;46:12065-72.

[29] Shen J, Huang W, Wu L, Hu Y, Ye M. The reinforcement role of different amino-functionalized multi-walled carbon nanotubes in epoxy nanocomposites. Compos Sci Technol 2007;67:3041-50.

[30] Ma PC, Kim JK, Tang BZ. Effects of silane functionalization on the properties of carbon nanotube/epoxy nanocomposites. Compos Sci Technol 2007;67:2965-72.

[31] Zaman I, Phan TT, Kuan HC, Meng Q, Bao La LT, Luong L, et al. Epoxy/ graphene platelets nanocomposites with two levels of interface strength. Polymer 2011;52:1603-11.

[32] Bugnicourt E, Galy J, Gérard JF, Barthel H. Effect of sub-micron silica fillers on the mechanical performances of epoxy-based composites. Polymer 2007;48:1596-605.

[33] Johnsen BB, Kinloch AJ, Mohammed RD, Taylor AC, Sprenger S. Toughening mechanisms of nanoparticle-modified epoxy polymers. Polymer 2007;48:530-41.

[34] Baselga J, Piérola IF, Serrano B, Pozuelo J, Cabanelas JC, Martín O. Fluorescence of polymers at interfaces: polymerization, relaxations, and imaging. Rev Fluoresc 2010;2010:311-47.

[35] Yang J, Sheng X, Zhang B, Fu R, Chen X. Intrinsic fluorescence studies of conformational relaxation and its dynamics of triblock copolymer 
during the micellization in selective solvents. Macromolecules 2011;44:1026-33.

[36] Valeur B. Molecular fluorescence. Principles and applications. 1st ed. Weinheim: Wiley-VCH; 2001.

[37] Lakowicz JR. Principles of fluorescence spectroscopy. 3rd ed. New York: Springer; 2006.

[38] Reichardt C. Solvatochromic dyes as solvent polarity indicators. Chem Rev 1994;94:2319-58.

[39] Loutfy RO. Fluorescence probes for polymer free-volume. Pure Appl Chem 1986;58:1239-48.

[40] Ellison CJ, Torkelson JM. Sensing the glass transition in thin and ultrathin polymer films via fluorescence probes and labels. J Polym Sci Part B Polym Phys 2002;40:2745-58.

[41] Ellison CJ, Torkelson JM. The distribution of glass-transition temperatures in nanoscopically confined glass formers. Nat Mater 2003;2:695-700.

[42] Priestley RD, Ellison CJ, Broadbelt LJ, Torkelson JM. Structural relaxation of polymer glasses at surfaces, interfaces, and in between. Science 2005;309:456-9.

[43] Rittigstein P, Torkelson JM. Polymer - nanoparticle interfacial interactions in polymer nanocomposites: confinement effects on glass transition temperature and suppression of physical aging. J Polym Sci Part B Polym Phys 2006;44:2935-43.

[44] Christoff M, Atvars TDZ. Phosphorescent probes in studies of secondary relaxation of amorphous polystyrene and poly(n-alkyl methacrylate)s. Macromolecules 1999;32:6093-101.

[45] Vigil MR, Bravo J, Atvars TDZ, Baselga J. Photochemical sensing of semicrystalline morphology in polymers: pyrene in polyethylene. Macromolecules 1997;9297:4871-6.

[46] Martins TD, Gulmine JV, Akcelrud L, Weiss RG, Atvars TDZ. Dependence of relaxation processes in a low-density polyethylene with different crosslink densities investigated by fluorescence spectroscopy. Polymer 2006;47:7414-24.

[47] Parker K, Schneider RT, Siegel RW, Ozisik R, Cabanelas JC, Serrano B, et al. Molecular probe technique for determining local thermal transitions: the glass transition at Silica/PMMA nanocomposite interfaces. Polymer 2010;51:4891-8.

[48] Li Y, Chan IL, Tyer L, Moody RT, Hirnel CM, Hercules DM. Study of solvents effects on the fluorescence of 1-(dimethylamino)-5-naphthalenesulfonic acid and related compounds. J Am Chem Soc 1975;97:3118-26.

[49] Ren B, Gao F, Tong Z, Yan Y. Solvent polarity scale on the fluorescence spectra of a dansyl monomer copolymerizable in aqueous media. Chem Phys Lett 1999;307:55-61.

[50] Hu Y, Horie K, Ushiki H. Fluorescence studies of the volume phase transition of poly(acrylamide) gels with a dansyl group. Macromolecules 1992:25:6040-4.

[51] Annaka M, Noda H, Motokawa R, Nakahira T. Fluorescence study on multiple phase behavior of dimethylacrylamide-methacrylic acid copolymer gel. Polymer 2001:42:09887-9894.

[52] Asano M, Winnik FM, Yamashita T, Horie K. Fluorescence studies of dansyl-labeled poly(N-isopropylacrylamide) gels and polymers in mixed water/methanol solutions. Macromolecules 1995;28:5861-6.

[53] Sarkar R, Ghosh M, Pal SK. Ultrafast relaxation dynamics of a biologically relevant probe dansyl at the micellar surface. J Photochem Photobiol B Biol 2005;78:93-8.

[54] Lochmüller CH, Marshall DB, Wilder DR. An examination of chemicallymodified silica surfaces using fluorescence spectroscopy. Anal Chim Acta 1981;130:31-43.

[55] Lenhart JL, van Zanten JH, Dunkers JP, Parnas RS. Interfacial response of a fluorescent dye grafted to glass. Langmuir 2000;16:8145-52.

[56] Gonzalez-Benito J, Mikes F, Bravo J, Aznar AJ, Baselga J. Fluorescence monitoring of curing process and water accessibility at glass fiber/epoxy interphase on composite materials. J Macromol Sci Phys 2001;B40:42941.

[57] Mikes F, Baselga J, Paz-Abuin S. Fluorescence probe-label methodology for in situ monitoring network forming reactions. Eur Polym J 2002;38:2393-404.

[58] Goda H, Frank CW. Fluorescence studies of the hybrid composite of segmented-polyurethane and silica. Chem Mater 2001;33:2783-7.

[59] Tsuneda S, Endo T, Saito K, Sugita K, Horie K, Yamashita T, et al. Fluorescence study on the conformational change of an amino groupcontaining polymer chain grafted onto a polyethylene microfiltration membrane. Macromolecules 1998;31:366-70.

[60] Cabanelas JC, Serrano B, Gonzalez-Benito J, Bravo J, Baselga J. Morphology of epoxy/polyorganosiloxane reactive blends. Macromol Rapid Commun 2001;22:694-9.
[61] El Harrak A, Carrot G, Oberdisse J, Eychenne-baron C, Boué F. Surfaceatom transfer radical polymerization from silica nanoparticles with controlled colloidal stability. Macromolecules 2004;37:6376-84.

[62] Zhuravlev LT. Concentration of hydroxyl groups on the surface of amorphous silicas. Langmuir 1987;3:316-8.

[63] Serrano B, Levenfeld B, Bravo J, Baselga J. Studies of polymerization of acrylic monomers using luminescence probes and differential scanning calorimetry. Polym Eng Sci 1996;36:175-81.

[64] Mikes F, Gonzalez-Benito F, Serrano B, Bravo J, Baselga J. Fluorescence monitoring of polymerization reaction. A new method for treating fluorescence experimental data. Polymer 2002;43:4331-9.

[65] Boens N, Qin W, Basaric N, Hofkens J, Ameloot M, Pouget J, et al. Fluorescence lifetime standards for time and frequency domain fluorescence spectroscopy. Anal Chem 2007;79:2137-49.

[66] Mittal KL, editor. Silanes and other coupling agents. Utrecht, The Netherlands: VSP; 1992.

[67] Kallury KMR, Macdonald PM, Thompson M. Effect of surface water and base catalysis on the silanization of silica by (aminopropyl) alkoxysilanes studied by X-ray photoelectron spectroscopy and $13 \mathrm{c}$ cross-polarization/magic angle spinning nuclear magnetic resonance. Langmuir 1994;10:492-9.

[68] Douce J, Boilot JP, Biteau J, Scodellaro L, Jimenez A. Effect of filler size and surface condition of nano-sized silica particles in polysiloxane coatings. Thin Solid Films 2004;466:114-22.

[69] Chen C, Justice RS, Schaefer DW, Baur JW. Highly dispersed nanosilica-epoxy resins with enhanced mechanical properties. Polymer 2008;49:3805-15.

[70] Kang S, Il S, Rim C, Park M, Rim S, Kim J. Preparation and characterization of epoxy composites filled with functionalized nanosilica particles obtained via sol-gel process. Polymer 2001;42:879-87.

[71] Kosower EM, Dodiuk H, Tanizawa K, Ottolenghi M, Orbach N Intramolecular donor-acceptor systems. Radiative and nonradiative processes for the excited states of 2-N-arylamino-6naphthalenesulfonates. J Am Chem Soc 1975;97:2167-78.

[72] Ding L, Kang J, Lü F, Gao L, Yin X, Fang Y. Fluorescence behaviors of 5-

dimethylamino-1-naphthalene-sulfonyl-functionalized selfassembled monolayer on glass wafer surface and its sensing properties for nitrobenzene. Thin Solid Films 2007;515:3112-9.

73] Ding L, Fang Y, Jiang L, Gao L, Yin X. Twisted intra-molecular electron transfer phenomenon of dansyl immobilized on chitosan film and its sensing property to the composition of ethanol-water mixtures. Thin Solid Films 2005;478:318-25.

[74] Ghiggino KP, Lee AG, Meech SR, O'Connor DV, Phillips D. Time-resolved emission spectroscopy of the dansyl fluorescence probe. Biochemistry 1981;20:5381-9.

[75] Zhong D, Pal SK, Zewail AH. Femtosecond studies of protein-DNA binding and dynamics: histone I. Chemphyschem 2001;2:219-27.

[76] Ellison CJ, Kim SD, Hall DB, Torkelson JM. Confinement and processing effects on glass transition temperature and physical aging in ultrathin polymer films: novel fluorescence measurements. Eur Phys J E 2002;8:155-66.

[77] Mundra MK, Ellison CJ, Behling RE, Torkelson JM. Confinement composition, and spin-coating effects on the glass transition and stress relaxation of thin films of polystyrene and styrene-containing random copolymers: sensing by intrinsic fluorescence. Polymer 2006;47:774759.

[78] Birks J. Photophysics of aromatic molecules. New York: WileyInterscience; 1970

[79] Israelachvili JN. Intermolecular and surface forces. 3rd ed. Academic Press; 2011.

[80] Van Den Berg O, Jager WF, Cangialosi D, Van Turnhout J, Verheijen PJT, $\mathrm{Wu} \mathrm{M}$, et al. A wavelength-shifting fluorescent probe for investigating physical aging. Macromolecules 2006;39:224-31.

[81] Donth E. The size of cooperative rearranging regions at the glass transition. J Non Cryst Solids 1982;53:325-30.

[82] Donth E. Characteristic length of the glass transition. J Polym Sci Part B Polym Phys 1996;34:2881-92.

[83] Fang M, Zhang $\mathrm{Z}$, Li J, Zhang $\mathrm{H}$, Lu H, Yang Y. Constructing hierarchically structured interphases for strong and tough epoxy nanocomposites by amine-rich graphene surfaces. J Mater Chem 2010;20:9635.

[84] Pistor V, Ornaghi FG, Ornaghi HL, Zattera AJ. Dynamic mechanical characterization of epoxy/epoxycyclohexyl-POSS nanocomposites. Mater Sci Eng A 2012;532:339-45.

[85] Srivastava S, Basu J. Experimental evidence for a new parameter to control the glass transition of confined polymers. Phys Rev Lett 2007;98:165701-1-4. 